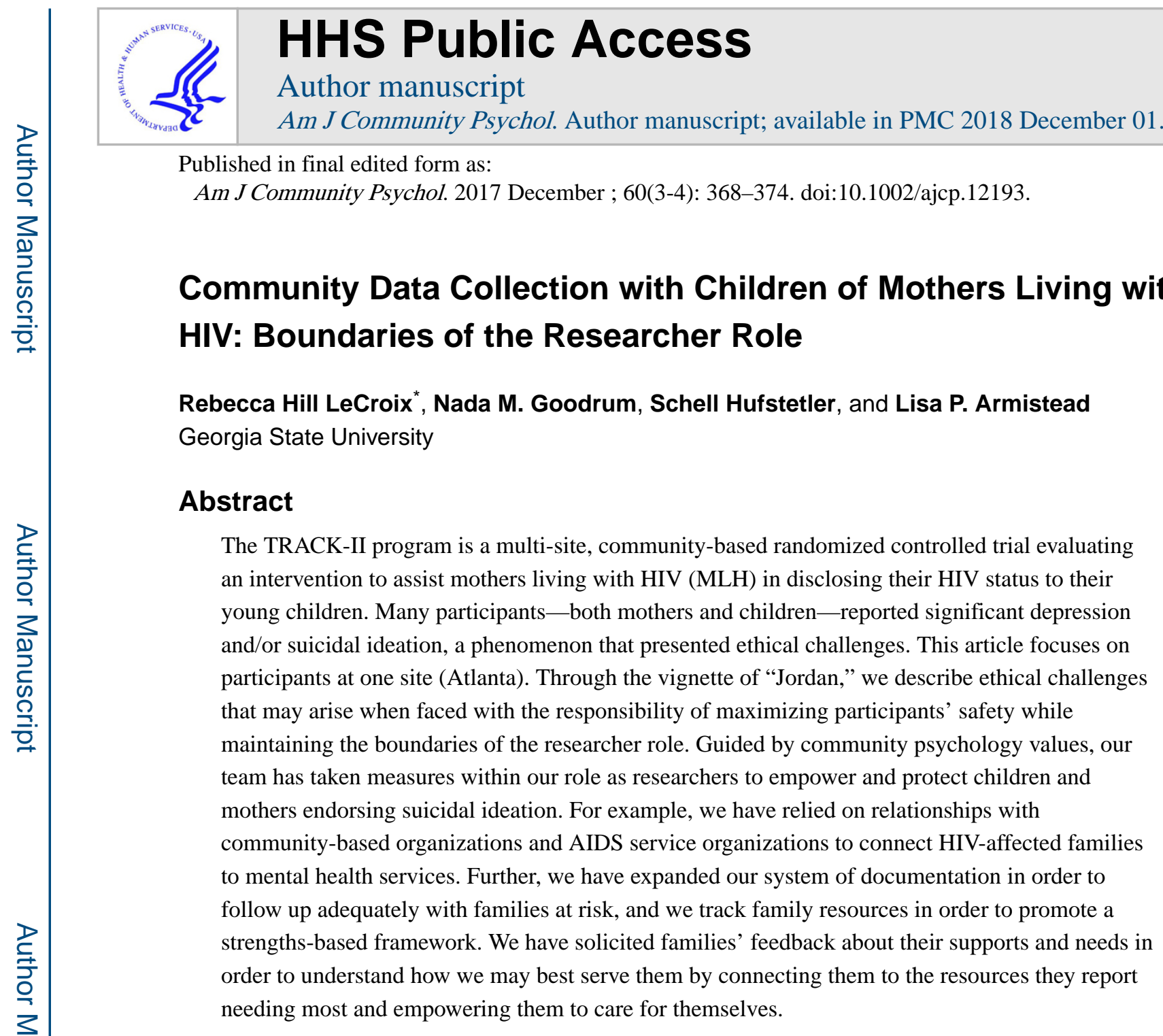

\title{
Keywords
}

HIV/AIDS; Ethical Issues in Research; Suicide; Families; Community-Based Research

\begin{abstract}
Working with vulnerable populations can present challenges for researchers. One challenge is the need to balance the principles of integrity and justice, as outlined in the American Psychological Association's Code of Ethics (APA, 2002). Psychologists should conduct research in a manner promoting "accuracy, honesty, and truthfulness" and must also "recognize that fairness and justice entitle all persons to access to and benefit from the contributions of psychology" (p.3). Balancing these principles comes into play when working with distressed research participants who do not have the means to secure psychological services. If an individual is at imminent risk for harm, the scale is clearly tipped in one direction - the researchers ensure that the individual receives care. For example, researchers may discover that a child is being maltreated and report this to authorities, even while understanding that reporting to child services will likely disrupt the
\end{abstract}

*Corresponding author. Department of Psychology, Georgia State University, P.O. Box 5010, Atlanta, GA 30302-5010; Phone: (404) 413-6315; rhlecroix@gmail.com. 
researcher-participant relationship. The balance is less clear when a participant is in distress, but not at imminent risk. For example, Olsen, Lehto, and Chan (2016) found that researchers working with end-of-life participants struggled to maintain the boundary of the researcher role, particularly when asked to provide medical care outside of the study. In this article we present a related challenge in the context of community-based research.

Community research often involves working with people in their home environment. However, this environment may lend itself to muddling of roles for the researchers. The Teaching, Raising, and Communicating with Kids (TRACK-II) Program focuses on understanding the ways in which mothers living with HIV (MLH) communicate with their children and aims to build upon the parent-child relationship to empower mothers in discussing their HIV status with their children. The vast majority of families enrolled in TRACK-II are socio-economically disadvantaged and reside in communities with very limited resources. This context combined with HIV-related stressors leads to emotional distress among many of our participants.

\section{Suicide and Depression among Children and Adolescents}

MLH may experience stress and anxiety related to their health status, which can compromise their parenting skills and in turn lead to negative child outcomes (e.g., Murphy, Marelich, Armistead, Herbeck, \& Payne, 2010). Mothers' physical and psychosocial health may contribute to children's depressive symptoms over time (Murphy, Marelich, \& Herbeck, 2012). Among children and adolescents in the general population, prevalence rates of depression range from 2.8\% to 10.6\% (Costello, Erkanli, \& Angold, 2006; Kessler, Petukhova, Sampson, Zaslavsky, \& Wittchen, 2012). Further, approximately $7.6 \%$ of persons 12 years of age and older suffer from depression in any given two-week period, and about 3\% of Americans in this age range have severe depressive symptoms (Pratt \& Brody, 2014). These rates of depression and suicidal ideation can be even higher among HIV-affected youth due to their mothers' compromised health, as well as the community contexts within which many HIV-affected families reside.

Participants in the TRACK-II project include mothers living with HIV who have children aged 6-14 who do not know about their mothers' HIV status. Following an initial baseline interview, half of the families are randomized to receive a three-session individual intervention. Intervention and control families also complete three follow-up interviews, at 3 months, 9 months, and 15 months. Interviews are conducted by trained research staff, including graduate students enrolled in clinical and community doctoral programs. Mothers in the intervention group receive three one-on-one sessions with a trained facilitator who leads the mothers through psychoeducational information about child development and assists in building communication skills and self-efficacy related to disclosure of the mother's HIV status to her child. Families in the waitlist control group receive the intervention in a group format after completion of the 15-month follow-up and mothers are not precluded from disclosing their HIV status to their children should they decide to do so throughout the course of the project. Participants are recruited at two sites, Atlanta and Los Angeles, and the project is supported through two NIH-funded grants (5R01MH094233 and 5R01MH094148) linked though the National Institutes of Health Collaborative R01 
mechanism. This manuscript is focused on the Atlanta site, which has enrolled 101 motherchild dyads.

During the ongoing TRACK-II study, mothers and children at the Atlanta site endorsed higher rates of suicidal ideation and depressive symptoms than seen in the general population. Approximately $15 \%$ of both mothers and children in the study reported depressive symptoms and/or suicidal ideation in the past two weeks, compared to $7.6 \%$ of adults (Pratt \& Brody, 2014) and 3-11\% of children in the general population (Costello et al., 2006; Kessler et al., 2012). Reports of depression and suicidal ideation from children as young as six years old can present ethical challenges related to finding appropriate and affordable clinical care referrals-rather than providing those services within the research context-and responsibility for ensuring that the family follows through with recommendations. The following vignette describes "Jordan," a 9-year-old boy whose story represents a blending of several participants from the Atlanta site.

\section{The Case of "Jordan"}

During the structured interview, researcher "Jane" asks Jordan to respond to the question from the Child Depression Inventory (Kovacs, 1985), "In the last two weeks, A) I do not think about killing myself; B) I think about killing myself but I would not do it; C) I want to kill myself." Jordan hesitates. Jane prompts, "Which would you pick about yourself, Jordan?"

Slowly, Jordan acknowledges thinking about suicide (Option B), but says, "I don't know that I would ever actually do it."

Jane begins a risk assessment: "Tell me what you've thought about, Jordan."

Jordan reports that the family has recently experienced several transitions. His mother moved the family from Georgia to North Carolina and then back to Georgia because of fear of a previous romantic partner who had been stalking the family. Jordan changed schools with each move and no longer feels confident in his school performance. According to Jordan, his mother has been very irritable lately, and he feels that she is unable to listen to his concerns. He reports his mother is his only family, and that, because of the family's multiple moves, he has been unable to keep long-term friendships. The family disruptions have left Jordan feeling hopeless and without social support, which has led to his consideration of suicide. He initially states that the easiest way to commit suicide would be to use a gun, and that it might be possible to procure one from a gun store. However, when Jane asks about the likelihood of obtaining a gun, Jordan acknowledges that it would be unlikely. He then notes access to a rope and the ability to climb onto the family's roof. He reports a plan for jumping from the roof with the rope around his neck. When asked to rate his intent to kill himself on a 0 (no intent) to 10 (full intent) scale, Jordan rates his intent as a 1 and states that he does not intend to follow through with his contemplated plans for suicide, because he expects things to improve since his family will stay in Atlanta.

After completing a thorough risk assessment and consulting with a supervisor, Jane reminds Jordan about the need to speak to his mother about his report of suicidal ideation and 
depressive symptoms. Jordan's mother was aware of these symptoms and has begun conversations with his school counselor. However, her own depressive symptoms and limited financial resources likely interfere with obtaining services for her child. Jane provides the family with a list of referrals and discusses the family's motivations and obstacles to pursuing counseling services for Jordan. Jane brings the family's struggles to the research team meeting to discuss any other potentially useful referrals and to process the interview and risk assessment. She follows up with a phone call to the family the following day to check in about the family's progress in contacting the referrals.

High-risk situations such as Jordan's highlight the sensitive balance between assessment for research purposes and mental health treatment, pushing us to consider the bounds of the role of researcher, particularly when children are involved. Families affected by HIV often experience additional stressors not directly related to HIV, such as poverty, discrimination, and limited access to resources (Murphy et al., 2010). Therefore, working with HIV-affected families necessitates a useful referral network. Unfortunately, even in large metropolitan areas like ours, low-cost referrals are often limited. Another ethical challenge is following up with the family after providing referrals - to what extent is the researcher responsible for ensuring that the family follows through?

\section{Researcher Roles and the Institutional Review Board}

When considering our legal and ethical responsibilities in this project, it has been important for us to define the bounds of our research role. Overall, our purpose in conducting this work is to reach valid and reliable conclusions about the efficacy of the TRACK-II intervention, while protecting and empowering the community with whom we work. Our role involves a wide range of procedural tasks, including recruiting participants by partnering with community agencies, scheduling baseline and follow-up interviews, obtaining informed consent and assent, tracking families' demographic and contact information, interviewing MLH and their children, facilitating an HIV disclosure intervention, disseminating results of our study, and other related tasks.

Beyond the project aims, our definition of the researcher role is guided by the Institutional Review Board, state law (e.g., mandated reporting of child abuse and neglect), and the APA Code of Ethics. Further, the socio-cultural context in which our participants are situated, as well as the mothers' HIV diagnosis, necessitate careful consideration of the boundaries of this role. Thus, our role encompasses providing referrals to families who are in need of mental health care services, and, in certain high-risk circumstances, providing additional protection as needed, such as the involvement of the Division of Family and Children Services and/or hospitalization for suicidal or homicidal risk. Importantly, though we have a responsibility to protect our participants by connecting them to resources, our role precludes functioning as psychotherapists (beyond the study intervention), case managers, or social workers for the families. For example, it would be inappropriate for interviewers to provide counseling services to Jordan and his family, despite the obvious need, even if the interviewers are qualified to do so. This boundary exists to prevent researchers from entering into multiple relationships with participants, which may—even inadvertently_lead to harm or exploitation of the participant (APA, 2002). 
Within the TRACK-II project, high-risk cases were expected given our target sample, though not at the level that has emerged. Based on the Principal Investigators' prior work with HIVaffected families, we anticipated that very few children would endorse suicidality, yet approximately one in seven children endorsed either thinking about suicide without intent or active suicidal ideation. As with all Human Subjects Research, our team relies on a research protocol approved by the relevant Institutional Review Boards (IRB): in our case, those at Georgia State University and the University of California, Los Angeles. The protocol involves obtaining informed consent (MLH) before the first interview and assent (children) before each interview, during which the role of the researcher is made clear. Additionally, the researcher must conduct a thorough risk assessment with participants (described below) who endorse any suicidal ideation, discuss with the child the need to have a conversation with the parent about the child's thoughts and/or behaviors, disclose the child's ideation to the parent, provide mental healthcare referrals, and consult with a licensed psychologist supervisor with a provision of psychiatric hospitalization if needed. It would be ideal, for example, for Jordan to feel comfortable enough to tell his mother about his suicidal thoughts and the precipitating events with support from the interviewer during this disclosure.

Subsequently, the interviewer would discuss potential resources and other sources of support for the family and encourage follow-up with these resources.

Many field procedures and strategies were useful in navigating risk assessment and management. One of the measures detailed in our IRB protocol to mitigate and manage risk is that the Computer Assisted Personal Interview (CAPI) software is programmed to alert the interviewer when a participant endorses suicidal ideation and/or clinical levels of depression. The interviewer then proceeds with a risk assessment. A thorough risk assessment involves gaining a detailed understanding of the individual's thoughts, feelings, and behaviors related to suicidality. In particular, this assessment includes evaluating the frequency, intensity, and duration of suicidal thoughts; content of these thoughts; past suicidal and self-harm behaviors; plans (as many as considered) for attempting suicide; intent to attempt suicide; reasons for considering suicide; and reasons for living.

Understanding a person's individual coping skills and their contextual resources (e.g., school counselor, low-cost clinics nearby, access to other resources and community support) gives a clear picture of strengths and needs. A thorough exploration of the person's situation and context, including internal experiences and risk and protective factors, allows the interviewer to provide more detailed and tailored resources for the situation. Fortunately, much of this information is gleaned through aspects of the structured interview used in data collection, which includes assessment of family relationships, social support, and self-esteem.

An appropriate risk assessment for the purposes of our project also includes attempting to connect the family to resources and empowering them to access care. Often, this may be included as part of a safety plan to be used in the event of a crisis. Of particular concern for our project is the limited resources available for low-income families. One of the ethical challenges we have faced is providing and following up with access to resources. Our TRACK-II team has generated a list of affordable mental health resources for adults, children, and families. To do so, we have relied on general knowledge of the community, consultation with other professionals in the counseling field, and suggestions of additional care sites as provided by community-based organizations (CBOs) and AIDS service 
organizations (ASOs) with which our team has formed relationships. Although our referral list initially included many HIV-specific resources and this list was provided only to mothers, we received feedback from the IRB indicating concerns that the listing of resources specific to HIV/AIDS may result in inadvertent disclosures to children regarding their mothers' HIV status. Based on our discussions with the IRB, we expanded the list to incorporate additional general community mental health resources. This expansion provided MLH with additional resources for their children who did not know about their HIV status, while maintaining MLH's privacy.

Although our team has an effective, IRB-approved safety protocol, our ethical challenge arises when the researchers' roles potentially expand to maximize participants' safety through connecting them to appropriate, available services without clinically intervening as researchers. Our researcher role encompassed follow-up calls and we did complete them to determine if families were able to connect with provided services. However, this role precluded us from paying for care or providing care ourselves; further, selectively providing care would violate the research protocol and potentially invalidate the valuable information gained from these participants. For example, our primary study aim is to evaluate the efficacy of an HIV disclosure intervention. If certain families in either the intervention or control condition receive additional services from the researchers, this may introduce a potential confound, reducing the internal validity of the study findings. Still somewhat morally vexing was the feeling that we could have enacted other procedures to assist families in receiving care. For example, knowing that Jordan's mother also experienced depression and related lack of motivation, Jane could have called the referrals with or for Jordan's mother rather than only providing the referral list. Additionally, as a majority of interviewers were students training in the helping professions, brief counseling could have been offered with the aim of improving mood and participants' motivation to seek care. Either step, but particularly the provision of clinical services, would be outside of the bounds of the researcher role and risked study integrity due to the potential of deviating from study protocol and introducing confounding variables.

\section{Resolution of Ethical Challenges}

Relationships with CBOs and ASOs have been crucial for empowering HIV-affected families with limited financial resources. The TRACK-II team initially did not anticipate a great need for resources in the community, based on prior experiences with these populations (e.g., the Principal Investigator's history of working with participants living with HIV), as well as the recruitment strategy of targeting ASOs. We expected that most families would be connected to care, because many of them were recruited from organizations providing care. Our proposal initially suggested that we would recruit $50 \%$ of our participants from a single ASO providing a comprehensive range of services to people living with HIV; however, only $8 \%$ of our sample was actually recruited from this site. Beyond other ASOs, recruitment was expanded to include agencies serving families with unstable housing and reliance on advertisements posted on public transportation. It is likely that the larger number of participants recruited from these methods resulted in a larger percentage of participants with depressive symptoms and suicidality. Additionally, mothers being connected to ASOs and other care providers did not guarantee that their children would have 
access to resources. In fact, most of the agencies with which the project staff interacted did not offer services to children of MLH.

Thus, with higher than expected rates of depressive symptoms and suicidal ideation among child participants, an ethical dilemma arose regarding our responsibility to provide adequate options for safety of our participants while maintaining a research, rather than healthcare provider, relationship with the families. In addition, our challenge included determining steps to assist families experiencing non-emergent distress who still needed quality psychological services. To resolve our ethical challenges, we further cultivated community relationships and relied upon additional referrals and consultations with others in the field. For instance, the institution where the project is based also houses a university psychology clinic. Through team members' relationships with this clinic, we found additional resources for low-income families and were able to refer all families needing any therapeutic services. Similarly, graduate student team members working in the community were able to solicit information from additional supervisors and community members working in, for example, nonprofit organizations, who had additional knowledge about community resources available for low-income families.

As we observed the relatively high rates of suicidal ideation among our participants, we expanded upon our review of associated documentation during team meetings in order to ensure adequate follow up with families at risk. To keep track of location and contact information about families enrolled in the program, our team uses Microsoft Access. This software is also equipped with an available "notes" section in which miscellaneous data can be included. In Jordan's case, Jane would have written a note including the risk item Jordan endorsed, current symptomology (including frequency, intensity, and duration of relevant symptoms and suicidal ideation), plans for self-harm and access to means, intent to kill himself (on a 0-10 scale) and his report of stressors, strengths and social supports. In addition, Jane would note Jordan's mother's current symptoms, Jane's consultation with the supervising psychologist, and the resource information provided to the family. In her notes, Jane may also include the family's level of engagement with current services or their intentions to connect with particular organizations or agencies. She would attempt to understand and communicate the family's current resources as well as their needs and preferences. Further, Jane would note if she provided any type of immediate clinical intervention (e.g., instilling hope) to encourage such contact. Also noted is additional follow up with the family provided by Jane or her supervisor to ascertain whether they were able to access mental health services or support. These details are then discussed at the weekly team meeting following the assessment and reviewed prior to the next follow up assessment.

This information is tracked not only for liability-related purposes, but, more importantly, to maintain a record of families that may need additional support in future interviews. Documentation allows interviewers to be more prepared for the possibility of a child or mother endorsing depressive symptoms or suicidal ideation and cues the interviewers about what has been done for this family in the past. This information is particularly important for interviewers new to the family. Our team attempts to maintain consistency with interviewers for each family; however, consistency is not always possible, based on schedules and interviewer transitions. Documentation allows interviewers to be aware of previous risk 
considerations for each family prior to follow-up interviews and promotes rapport and a trusting relationship with the family, even when interviewers change over time. An additional benefit of this practice is that we are able to track families' strengths and resources in each family, not only those at high risk, so that our framework over time has shifted to be more focused on empowerment and strengths-based relationship building and focused less on deficits and lack of resources.

\section{Community Psychology Values Informing Ethical Responses}

Community psychology values have guided the ways the research team has approached ethical challenges in our work with children from underserved communities. Specifically, the choice to focus on a historically disenfranchised group with an aim of building empowerment (Kloos et al., 2012) has been important throughout the TRACK-II project. During the conceptualization of this project, the team often viewed its development from a community perspective, as originally proposed by Sarason (1976), rather than a theory related to individuals. For example, interviews are typically conducted in participants' homes or other location of their choice to increase their ownership of and comfort with the project (Levine, Perkins, \& Perkins, 2005). Whenever an ethical challenge has arisen, the research team first has a conversation about possible avenues to resolve the conflict, always with the family's wellness, health, and strengths in mind (Levine et al., 2005). Consistent documentation and weekly research team meetings support these efforts.

The TRACK-II project is influenced by the perspective that people's social environments influence their access to health and wellbeing (Levine et al., 2005). Thus, the TRACK-II team has conceptualized families' struggles as products of their environments and the mothers' compromised health, including a very limited health support infrastructure. Overall, our ethical challenges, relating to our roles as researchers in the community interviewing children and adolescents endorsing depression and suicidal ideation, revolved around our responsibility to assist in connecting the families to appropriate resources (as defined by the family and to maximize their sense of community; Sarason, 1976), while not undertaking the role of mental health care provider with the family, despite significant participant distress. These challenges were addressed in part through our team's connection to community psychology values. In particular, the appreciation of and focus on empowerment, consultation, and strengths allowed the team to consider ways to help families build upon the supports already available to them rather than assuming their needs (Kloos et al., 2012). Further, our efforts to build collaborative community relationships ("networks," per Sarason, 1976) have been critical to the process. Without valuing these relationships, we could not help foster relationships between TRACK-II participants and CBOs/ASOs and would face an even greater challenge in regard to our roles as researchers, not clinicians.

\section{Lessons Learned}

TRACK-II is in its fifth year, and our work with families and community partners has resulted in many lessons, some of which continue to shape the ways we work with families affected by HIV in the Atlanta community. Most prominently, the value of collaborative 
community relationships has been reinforced many times throughout the project. Without such collaborations, we would not have been able to recruit, retain, or refer to resources the majority of our participants. We would have very few suggestions for families asking for assistance and even fewer resources for children endorsing high levels of depressive symptoms or suicidal ideation.

The project has also been instructive with respect to the value of consultation and supervision. Many of the research interviewers are clinical and community psychology graduate students at this Atlanta site, and learning to assess risk associated with suicidal ideation, particularly with children, can be a difficult process. Self-reflection and processing of interviewer experiences has been invaluable throughout this project, and the input and guidance of more experienced researchers, clinicians, and community advocates have allowed for learning and growth for the students. The students are better equipped to work with disenfranchised families when supported by an encouraging and empathic team and supervisor. The creation of a supportive team atmosphere allowed ethical challenges to be presented and discussed while resolution is reached in a purposefully cultivated collaborative climate.

Lastly, an important lesson from these experiences includes the need for child-focused mental health resources. Until this project was fully underway and children like Jordan began reporting depressive symptoms and suicidal ideation, the lack of resources had not been highlighted so dramatically to the research team. It is well understood that services for children are limited; however, the implementation of this project has emphasized the need for more structural and systemic focus on behavioral health disorders and concerns for children, at least in the metropolitan Atlanta area and particularly for children in families experiencing stressors such as HIV.

Community psychology values inform the ways in which we have worked and continue to work through these ethical challenges. The TRACK-II research team collaborates with community-based organizations not only to recruit and retain families, but also to seek available resources and to connect families in underserved communities to these resources. Going beyond providing referrals, we seek to empower these families to help themselves by advocating for their needs with community organizations. The work is made possible for the (primarily) graduate student researchers through the advisement and supervision of a doctoral-level psychologist. By working with the families to understand their needs and preferences, we are better able to empower them to connect to services that enable families to flourish.

\section{Acknowledgments}

Research reported in this manuscript was supported by the National Institute of Mental Health of the National Institutes of Health by Grant Numbers 5R01MH094233 (PI: Lisa P. Armistead) and 5R01MH094148 (PI: Marya T. Schulte). The content is solely the responsibility of the authors and does not necessarily represent the official views of the National Institutes of Health. Conflict of Interest: The authors declare that they have no conflict of interest. 


\section{References}

American Psychological Association. Ethical principles of psychologists and code of conduct. American Psychologist. 2002; 57:1060-1073. [PubMed: 12613157]

Costello JE, Erkanli A, Angold A. Is there an epidemic of child or adolescent depression? Journal of Child Psychology and Psychiatry and Allied Disciplines. 2006; 47:1263-1271. DOI: 10.1111/j. 1469-7610.2006.01682.x

Kloos, B., Hill, J., Thomas, E., Wandersman, A., Elias, MJ., Dalton, JH. Community psychology: Linking individuals and communities. 3. Belmont, CA: Wadsworth; 2012.

Kessler RC, Petukhova M, Sampson NA, Zaslavsky AM, Wittchen HU. Twelve-month and lifetime prevalence and lifetime morbid risk of anxiety and mood disorders in the United States. International Journal of Methods in Psychiatric Research. 2012; 21:169-184. DOI: 10.1002/mpr. 1359 [PubMed: 22865617]

Kovacs M. The Children's Depression Inventory (CDI). Psychopharmacology Bulletin. 1985; 21:995998. [PubMed: 4089116]

Levine, M., Perkins, DD., Perkins, DV. Principles of community psychology: Perspectives and applications. 3. New York, NY: Oxford University Press; 2005.

Murphy DA, Marelich WD, Armistead L, Herbeck DM, Payne DL. Anxiety/stress among mothers living with HIV: Effects on parenting skills and child outcomes. AIDS Care. 2010; 22:1449-1458. DOI: 10.1080/09540121.2010.487085 [PubMed: 20824552]

Murphy DA, Marelich WD, Herbeck DM. Impact of maternal HIV health: A 12-year study in the Parents and Children Coping Together project. Journal of Adolescent Health. 2012; 51:313-318. [PubMed: 22999830]

Olsen DP, Lehto RH, Chan RR. Ethical case study of the researcher-participant relationship in end-oflife research. Western Journal of Nursing Research. 2016; 38:1205-1220. DOI: 10.1177/0193945916639590 [PubMed: 27006191]

Pratt, LA., Brody, DJ. Centers for Disease Control and Prevention NCHS Data Brief, No. 172. 2014. Depression in the U. S. household population, 2009-2012.

Sarason SB. Community psychology, networks, and Mr. Everyman. American Psychologist. 1976; 31:317-328. [PubMed: 1008335] 